\title{
POST'S PROBLEM, ADMISSIBLE ORDINALS, AND REGULARITY
}

\author{
BY \\ GERALD E. SACKS(1)
}

1. Introduction. The basic notions of metarecursion theory were introduced in [7]. Metarecursion theory is an attempt to generalize the ideas and arguments of recursion theory from the natural numbers to the recursive ordinals. Ordinary recursion theory concerns itself with finite sets of natural numbers. Metarecursion theory deals in an analogous fashion with metafinite sets of recursive ordinals. In [7] it was seen that two of the deepest results of ordinary recursion theory, the solution of Post's problem [3] and the maximal set construction [4], generalize to the metarecursive case. Theorem 4 of [7] states that there exist two metarecursively enumerable sets of recursive ordinals such that neither is metarecursive in the other. Kreisel [6] casts doubt on the contention that Theorem 4 of [7] is the correct generalization of Post's problem. He wonders how to correctly formulate the notion of Turing reducibility for recursive ordinals and he discusses four possible choices. It is not our place to decide the correct notion of Turing reducibility for metarecursion theory; however, in $\$ 4$ we show that there exist two metarecursively enumerable sets such that neither is reducible to the other by any of the four methods discussed by Kreisel.

In [7] two sets of recursive ordinals are said to have the same metadegree if each is metarecursive in the other. It is not known if the metadegrees of metarecursively enumerable sets are order-isomorphic to the Turing degrees of recursively enumerable sets, but all the existing evidence seems to favor an affirmative answer. Driscoll [2] has shown the metadegrees of metarecursively enumerable sets are dense; i.e., between any two comparable ones, there is a third. His proof is not a mere translation of the argument of [12]. There exists a formidable obstacle to the lifting up into metarecursion theory of results about recursively enumerable sets. Call a set of recursive ordinals regular if its intersection with every metafinite set is metafinite. In ordinary recursion theory, the intersection of any set of natural numbers with any finite set is finite; this platitude is essential in many arguments about recursively enumerable sets. Unfortunately, there exists a multitude of nonregular, metarecursively enumerable sets [7].

Received by the editors September 28, 1965 and, in revised form, February 8, 1966.

(1) The preparation of this paper was supported by U.S. Army Contract ARO-D-373. The author wishes to thank Professor G. Kreisel for invaluable help in the writing of this paper. 
Nonetheless, Driscoll [2] was able to make a long detour around the obstacle of nonregularity with the aid of the principal result of $\$ 2$ : each metarecursively enumerable set has the same metadegree as some regular, metarecursively enumerable set.

Spector [14] showed that all nonhyperarithmetic, $\Pi_{1}^{1}$ sets have the same hyperdegree. It is possible to interpret his result as the answer to Post's problem for $\Pi_{1}^{1}$ sets. In this paper we offer what we hope is a happier alternative. Suppose we identify the natural numbers with the finite, recursive ordinals. Then, as was seen in [7], the $\Pi_{1}^{1}$ sets of natural numbers coincide with the metarecursively enumerable sets of finite, recursive ordinals. In $\$ 3$ we show that there exist two $\Pi_{1}^{1}$ sets of natural numbers such that neither is metarecursive in the other.

Kripke [8], [9] has succeeded in generalizing a large portion of recursion theory by introducing the notion of admissible ordinal. An admissible ordinal has the property that the set of all lesser ordinals behaves very much like the natural numbers when one attempts to initiate the pre-Friedbergian arguments of recursion theory. Kripke's definition of admissible ordinal is expressed in terms of closure properties that refer to an equation calculus analogous to the one employed by Kleene [5] for ordinary recursion theory. Kripke's calculus is very convenient for the purposes of metarecursion theory and was utilized in [7]. (It turns out that $\omega_{1}$, the least nonrecursive ordinal, is the first admissible ordinal occurring after $\omega$.) In this paper, our first duty is metarecursion theory, but we cannot resist indicating which of our results generalize to every admissible ordinal. In particular, we give a solution to Post's problem which is uniform with respect to all admissible ordinals; i.e., we give a single argument in which the admissible ordinal $\alpha$ occurs as a parameter.

We conclude with a discussion of some open questions.

2. Regularity and metarecursive enumerability. In [7] a set of recursive ordinals was said to be regular if its intersection with every metafinite set was metafinite. Each metarecursive set is regular. Each nonhyperarithmetic, $\Pi_{1}^{1}$ set of natural numbers (regarded as a set of finite, recursive ordinals) is a nonregular, metarecursively enumerable set. In metarecursion theory we are forced to deal with bounded, metarecursively enumerable sets which are not metarecursive. This last remark is the reason that the phenomenon of nonregularity makes it difficult, if not impossible, to lift many results of ordinary recursion theory up into metarecursion theory. But the situation is not as gloomy as it appears, since we can show by an essentially elementary argument that each metadegree of a metarecursively enumerable set contains a regular, metarecursively enumerable set. In $\$ 4$ we see that there exist metadegrees of metarecursively enumerable sets which contain only regular sets. The overall situation concerning regularity is not clear and is discussed in $\$ 7$. 
THEOREM 1. Each metarecursively enumerable set has the same metadegree as some regular, metarecursively enumerable set.

Proof. The main idea of what follows is inspired by Dekker's notion of deficiency set [1]. We use what might be called a double deficiency set. Let $\left\{K_{\alpha}\right\}$ be a standard indexing of the metafinite sets; i.e., there exist metarecursive functions $j$ and $k$ such that for each $\alpha, K_{\alpha}=\{\beta \mid \beta<j(\alpha)$ and $k(\alpha, \beta)=0\}$. Let $Q$ be a $\Pi_{1}^{1}$ set of unique notations for the recursive ordinals; let $\eta$ be a metarecursive function such that for each $\alpha, \eta(\alpha)$ is the unique natural number in $Q$ which is a notation for $\alpha$. For convenience we assume that $Q$ is a linearly ordered subset of Kleene's 0 .

We write $A \leqq_{M} B$ to indicate $A$ is metarecursive in $B$. Intuitively, $A \leqq_{M} B$ means there exists a method $M$ for determining the truth or falsity of both of the following two assertions for an arbitrary metafinite $K: K \subseteq A$ and $K \subseteq c A$; the method $M$ must be such that the status of $K$ is decided by the fact that $B$ satisfies some metafinite set of conditions such as those expressed by $K_{1} \subseteq B$ and $K_{2} \subseteq c B$. Thus $M$ can be thought of as a metarecursively enumerable set of 5-tuples of the form $\left\langle 0, K, K_{1}, K_{2}, i\right\rangle$ and $\left\langle 1, K, K_{1}, K_{2}, i\right\rangle$, where $K \subseteq A$ if and only if for some $K_{1} \subseteq B$ and $K_{2} \subseteq c B$, we have $\left\langle 0, K, K_{1}, K_{2}, 0\right\rangle \in M$, where $K \nsubseteq A$ if and only if for some $K_{1} \subseteq B$ and $K_{2} \subseteq c B$, we have $\left\langle 0, K, K_{1}, K_{2}, 1\right\rangle \in M$, and where a similar relation holds between the 5-tuples of the form $\left\langle 1, K, K_{1}, K_{2}, i\right\rangle, c A$, and $B$.

We write $A \leqq_{m} B$ (read $A$ is many-one reducible to $\left.B\right)$ to indicate that for some metarecursive function $f$, we have $\alpha \in A \leftrightarrow f(\alpha) \in B$. If $A \leqq_{m} B$, then $A \leqq_{M} B$. We write $A \leqq_{w} B$ to indicate $A$ is weakly metarecursive in $B$. Let $F_{\alpha}$ be a standard enumeration of all the finite sets of recursive ordinals. We write $A \leqq{ }_{f} B(\operatorname{read} A$ is finitely reducible to $B$ ) to indicate $A \leqq_{w} B$ (there is an $e$ such that $A=\{e\}^{B}$ ) and there is a metarecursive $g$ such that for each $\alpha$, the computation of the truth value of $\alpha \in A$ draws only on the information expressed by the membership of $F_{g(\alpha)} \subseteq B$ and $F_{g(\alpha)} \subseteq c B$. If $A \leqq_{f} B$ and $B \leqq_{w} C$, then $A \leqq_{w} C$. We need the notion of finite reducibility because Driscoll [2] has shown that the relation $\leqq_{w}$ is not transitive.

Suppose $A$ is metarecursively enumerable and we wish to prove $A \leqq_{M} B$. It is sufficient to find a method $M$ for showing $K \subseteq c A$ when and only when $K \subseteq c A$ is true. We merely combine the method $M$ with an enumeration of $A$. If $K \subseteq A$, then eventually we will enumerate all of $K$ in $A$. If $K \nsubseteq A$, then $M$ will show that for some $\alpha \in K$, we have $\{\alpha\} \subseteq c A$. If $K \subseteq c A$, then $M$ will show $K \subseteq c A$. If $K \nsubseteq c A$, then for some $\alpha \in K$, we will cnumerate $\alpha \in A$. We will refer to the above observation as principle $\mathrm{P}$.

Let $A$ be a metarecursively enumerable set. We aim to find a metarecursively enumerable, regular $B$ in the same metadegree as $A$. Let $A^{*}=\left\{\alpha \mid A \cap K_{\alpha} \neq \varnothing\right\}$. We check that $A$ and $A^{*}$ have the same metadegree. Observe that

$$
K_{\alpha} \subseteq c A^{*} \leftrightarrow \bigcup\left\{K_{\gamma} \mid \gamma \in K_{\alpha}\right\} \subseteq c A \leftrightarrow K_{h(\alpha)} \subseteq c A \leftrightarrow h(\alpha) \in c A^{*},
$$


where $h$ is metarecursive. (We are using very heavily the fact that a metafinite union of metafinite sets is metafinite.) It follows from principle $\mathbf{P}$ and (1) that $A^{*}$ is metarecursive in $A$. The definition of $A^{*}$ implies that $A$ is metarecursive in $A^{*}$.

Let $f$ be a one-one, metarecursive function whose range is $A^{*}$. We introduce the double deficiency set of $f$ :

$$
D_{f}^{2}=\{\alpha \mid(E \beta)(f(\beta)<f(\alpha) \& \eta(f(\beta))<\eta(f(\alpha)) \& \beta>\alpha)\} .
$$

We show $D_{f}^{2}$ is regular by showing $D_{f}^{2} \cap L_{\gamma}$ is metafinite for every $\gamma$ $\left(L_{\gamma}=\{\alpha \mid \alpha<\gamma\}\right)$. Fix $\gamma$; we need only find a $\tau$ such that

$$
D_{f}^{2} \cap L_{\gamma}=\left\{\alpha \mid(E \beta)_{\beta<\tau}(f(\beta)<f(\alpha) \& \eta(f(\beta))<\eta(f(\alpha)) \& \beta>\alpha)\right\} .
$$

We define a partial metarecursive function $d$ by induction:

$$
\begin{gathered}
d(0) \cong \mu \beta(\beta>\gamma \&(E \alpha)(\alpha<\gamma \& f(\beta)<f(\alpha) \& \eta(f(\beta))<\eta(f(\alpha)))) ; \\
d(n+1) \cong \mu \beta\left(\beta>d(n) \&(m)_{m \leqq n}(f(\beta)<f(d(m)) \bigvee \eta(f(\beta))<\eta(f(d(m))))\right. \\
\&(E \alpha)(\alpha<\gamma \& f(\beta)<f(\alpha) \& \eta(f(\beta)<\eta(f(\alpha)))) .
\end{gathered}
$$

Clearly, the domain of $d$ is a finite, initial segment of the natural numbers. Let $\tau$ be the least ordinal greater than $\gamma$ and greater than any element of the range of $d$. Then $\tau$ satisfies (2). Note that $\tau$, regarded as a function of $\gamma$, is weakly metarecursive in $A^{*}$. Then it follows from (3) and principle $P$ that $D_{f}{ }^{2}$ is metarecursive in $A^{*}$.

$$
K_{\alpha} \subseteq c D_{f}^{2} \leftrightarrow K_{\alpha} \cap D_{f}^{2} \cap L_{j(\alpha)}=\varnothing
$$

(Recall that $K_{\alpha} \subseteq L_{j(\alpha)}$ and $j$ is metarecursive.)

Now we make an assumption about $D_{f}^{2}$ and show $A^{*}$ is metarecursive in $D_{f}^{2}$. Let $\rho$ be defined by

$$
\rho(\alpha) \cong \mu \beta\left(f(\beta)>\alpha \& \eta(f(\beta))>\eta(\alpha) \& \beta \notin D_{f}^{2}\right) .
$$

We assume $\rho$ is total. Then $\rho$ is weakly metarecursive in $D_{f}^{2}$, and (1) tells us

$$
K_{\alpha} \subseteq c A^{*} \leftrightarrow h(\alpha) \notin A^{*} \leftrightarrow h(\alpha) \notin\{f(\beta) \mid \beta<\rho(h(\alpha))\} .
$$

Finally, we are faced with the problem that $\rho$ may not be total. Let $\gamma$ be the least ordinal such that

$$
(\beta)\left(f(\beta) \leqq \gamma \vee \eta(f(\beta)) \leqq \eta(\gamma) \vee \beta \in D_{f}^{2}\right)
$$

Then there must be a $\sigma$ such that $\eta(f(\beta))>\eta(\gamma)$ for all $\beta>\sigma$, and

$$
(\beta)_{\beta>\sigma}\left(f(\beta) \leqq \gamma \vee \beta \in D_{f}^{2}\right)
$$


Let $A_{\gamma}{ }^{*}=A^{*} \cap L_{\gamma}$. We claim $A_{\gamma}{ }^{*}$ and $A^{*}$ have the same metadegree. Let $A^{\prime}=A^{*}-\left\{\alpha \mid \alpha \leqq \gamma \vee(E \delta)_{\delta \leqq \sigma}(\alpha=f(\delta))\right\}$. It suffices to show $A^{\prime}$ is metarecursive in $A_{\gamma}{ }^{*}$. We make repeated applications of (5):

$$
\begin{gathered}
\alpha \in A^{\prime} \leftrightarrow(E \beta)_{\beta>\sigma}\left(f(\beta)=\alpha \& \beta \in D_{f}^{2}\right), \\
\beta \in D_{f}^{2} \leftrightarrow\left(E \delta_{1}\right)\left(\delta_{1}>\beta \& f\left(\delta_{1}\right)<\alpha \& \eta\left(f\left(\delta_{1}\right)\right)<\eta(\alpha)\right), \\
\delta_{1}>\sigma \& f\left(\delta_{1}\right)>\gamma \rightarrow \delta_{1} \in D_{f}^{2}, \\
\delta_{1} \in D_{f}^{2} \leftrightarrow\left(E \delta_{2}\right)\left(\delta_{2}>\delta_{1} \& f\left(\delta_{2}\right)<f\left(\delta_{1}\right) \& \eta\left(f\left(\delta_{2}\right)\right)<\eta\left(f\left(\delta_{1}\right)\right)\right), \\
\delta_{2}>\sigma \& f\left(\delta_{2}\right)>\gamma \rightarrow \delta_{2} \in D_{f}^{2}, \\
\vdots \\
\left(E \delta_{m}\right)\left(f\left(\delta_{m}\right) \leqq \gamma \& \eta\left(f\left(\delta_{m}\right)\right)<\eta(\alpha)\right) .
\end{gathered}
$$

But then we have

$$
\begin{gathered}
\alpha \in A^{\prime} \leftrightarrow(E \beta)(E \delta)(\sigma<\beta<\delta \& \alpha=f(\beta) \\
\& f(\delta) \leqq \gamma<\alpha \& \eta(f(\delta))<\eta(\alpha)) .
\end{gathered}
$$

Let $F_{t(\alpha)}$ be the finite set $\{\varepsilon \mid \varepsilon \leqq \gamma \& \eta(\varepsilon)<\eta(\alpha)\}$; we can assume $t$ is metarecursive, since our unique notations form a linearly ordered subset of Kleene's 0 . Then (6) becomes

$$
\alpha \in A^{\prime} \leftrightarrow(E \beta)(E \delta)\left(\sigma<\beta<\delta \& \gamma<\alpha=f(\beta) \& f(\delta) \in F_{t(\alpha)}\right) .
$$

With the help of (1) we obtain

$$
K_{\alpha} \subseteq c A^{\prime} \leftrightarrow\left(K_{\alpha}-\left(A^{*}-A^{\prime}\right)\right) \subseteq c A^{*} \leftrightarrow q(\alpha) \in c A^{\prime},
$$

where $q$ is metarecursive (we assume some repetition in our indexing of the metafinite sets). Principle P, (7) and (8) imply $A^{\prime}$ is metarecursive in $A_{\gamma}{ }^{*}$.

Let $B=\left\{\eta(\alpha) \mid \alpha \in A_{\gamma}{ }^{*}\right\}$. Since $A_{\gamma}{ }^{*}$ is bounded, it must be that $B$ and $A_{\gamma}{ }^{*}$ have the same metadegree; in fact, $B \leqq_{m} A_{\gamma}{ }^{*}$ and $A_{\gamma}{ }^{*} \leqq_{m} B$. $B$ is useful because it contains only finite ordinals. Let $g$ be a one-one, metarecursive function whose range is $B$. Let

$$
D_{g}=\{\alpha \mid(E \beta)(g(\beta)<g(\alpha) \& \beta>\alpha)\} .
$$

$D_{g}$ is seen to be regular by the same argument we used to see $D_{f}^{2}$ is regular.

We claim $D_{g}$ and $A_{\gamma}{ }^{*}$ have the same metadegree. $D_{g}$ is metarecursive in $B$, and hence in $A_{\gamma}{ }^{*}$, by the same argument used to show $D_{f}{ }^{2}$ is metatecursive in $A^{*}$. It remains only to show $A_{\gamma}{ }^{*}$ is metarecursuve in $D_{g}$. We note 


$$
\begin{gathered}
K_{\alpha} \subseteq c A_{\gamma}{ }^{*} \leftrightarrow(\tau)\left(\tau \in K_{\alpha} \& \tau<\gamma \rightarrow K_{\tau} \cap A=\varnothing\right) \\
\leftrightarrow K_{m(\alpha)} \subseteq c A \leftrightarrow h(m(\alpha)) \in c A^{*},
\end{gathered}
$$

where $m$ is metarecursive. We can choose $h$ and $m$ so that

$$
(\alpha)\left(h(m(\alpha))>\gamma \& \eta(h(m(\alpha))>\eta(\gamma)) \&(\delta)_{\delta>\sigma}(h(m(\alpha)) \neq f(\delta))\right) .
$$

Then we have

$$
K_{\alpha} \subseteq c A_{\gamma}{ }^{*} \leftrightarrow h(m(\alpha)) \in c A^{\prime} .
$$

Now $B \leqq_{w} D_{g}$ by an argument similar to the one used to show $A^{*} \leqq_{M} D_{f}{ }^{2}$. (Actually, this is the argument used by Dekker [1] to prove the range of a one-one, recursive function is recursive in its deficiency set.) But then $A_{\gamma}{ }^{*} \leqq_{w} D_{g}$, since $A_{\gamma}{ }^{*} \leqq_{m} B$. It follows from (7) that $A^{\prime} \leqq_{f} A_{\gamma}{ }^{*}$; consequently, $A^{\prime} \leqq_{w} D_{g}$. This last together with (11) and principle P give $A_{\gamma}{ }^{*} \leqq{ }_{M} D_{g}$.

The proof of Theorem 1 suffers from a certain lack of uniformity; this defect is discussed in $\$ 7$.

Let $\alpha$ be an arbitrary admissible ordinal [8], [9]. Kripke calls a set of ordinals $\alpha$-finite if it is $\gamma$-recursive and is bounded by some ordinal less than $\alpha$. Call a set of ordinals (less than $\alpha$ ) regular if its intersection with every $\alpha$-finite set is $\alpha$-finite. Define the notion of " $\alpha$-recursive in" by strict analogy with the notion of "metarecursive in" as defined in [7]; simply replace "metarecursive" by " $\alpha$-recursive" and "metafinite" by " $\alpha$-finite". Let us say two sets have the same $\alpha$-degree if each is $\alpha$-recursive in the other.

THEOREM $1^{\alpha}$. Each $\alpha$-recursively enumerable set has the same $\alpha$-degree as some regular, $\alpha$-recursively enumerable set.

Proof. We proceed as in Theorem 1, but some modifications are necessary. Let $\alpha^{*}$ be the projectum of $\alpha$. (Kripke defines the projectum of $\alpha$ to be the least ordinal $\beta$ such that there exists a one-one, $\alpha$-recursive function whose range is bounded by $\beta$.) Note that an $\alpha$-recursively enumerable set bounded by an ordinal less than $\alpha^{*}$ must be $\alpha$-finite; this fact is analogous to the truism that every finite set of natural numbers is metafinite. Let $t$ be a one-one, $\alpha$-recursive function whose values are less than $\alpha^{*}$. Then $t(\alpha)$ plays the same role that $\eta(\alpha)$ did in the proof of Theorem 1. The only difficulty lies in showing that $A^{\prime}$ is $\alpha$-recursive in $A_{\gamma}^{*}$ and that $A_{\gamma}^{*}$ is $\alpha$-recursive in $D_{g}$. Equation (6) becomes

$$
\eta \in A^{\prime} \leftrightarrow(E \beta)(E \delta)(\sigma<\beta<\delta \& \eta=f(\beta) \& f(\delta) \leqq \gamma<\eta \& t(f(\delta))<t(\eta)) .
$$

Let $K_{s(\eta)}$ be the $\alpha$-finite set $\{\varepsilon \mid \varepsilon \leqq \gamma \& t(\varepsilon)<t(\eta)\}$, where $s$ is $\alpha$-recursive.

$$
\eta \in A^{\prime} \leftrightarrow(E \beta)(E \delta)\left(\sigma<\beta<\delta \& \gamma<\eta=f(\beta) \& f(\delta) \in K_{s(\eta)}\right) .
$$

The set $\left\{t(f(\delta)) \mid f(\delta) \in K_{s(\eta)}\right\}$ is $\alpha$-finite, since it is $\alpha$-recursively enumerable and bounded by $t(\eta)<\alpha^{*}$. But then the set $\left\{f(\delta) \mid{ }^{f}(\delta) \in K_{s(\eta)}\right\}$ is $\alpha$-finite. It follows from $\left(7^{\alpha}\right)$ and (8) that $A^{\prime}$ is $\alpha$-recursive in $A_{\gamma}{ }^{*}$. The computation of the truth- 
value of $\eta \in A^{\prime}$ from $A_{\gamma}^{*}$ uses only information about the membership of $A_{\gamma}^{*}$ involving ordinals $\varepsilon$ such that $t(\varepsilon)<t(\eta)$. But the computation of the truth-value of $\eta \in A^{\prime}$ from $B$ uses only information about the membership of $B$ involving ordinals less than $t(\eta)<\alpha^{*} . D_{g}$ is not $\alpha$-finite, so there must be a $\tau$ such that $g(\tau)>t(\eta)$ and $\tau \notin D_{g}$. Any member of $B$ less than $t(\eta)$ must be enumerated by $g$ before $g(\tau)$. Thus the computation of the truth-value of $\eta \in A^{\prime}$ from $D_{g}$ is accomplished using only finitely much information about the membership of $D_{g}$. In short, $A^{\prime}$ is weakly $\alpha$-recursive in $D_{g}$. This last together with (11) and principle $\mathrm{P}$ gives $A_{\gamma}{ }^{*}$ is $\alpha$-recursive in $D_{g}$.

We can strengthen Theorem $1^{\alpha}$ as follows. Let $\alpha$ be an admissible ordinal. Let $c B$, the complement of $B$, denote $\{\beta \mid \beta<\alpha\}-B$. Call an $\alpha$-recursively enumerable set unbounded if it is not bounded by any ordinal less than $\alpha$. We say an $\alpha$-recursively enumerable set $B$ is simple if $c B$ is unbounded, and if every $\alpha$-recursively enumerable subset of $c B$ is $\alpha$-finite. Then we can prove: each non- $\alpha$-recursive, $\alpha$-recursively enumerable set has the same $\alpha$-degree as some regular, simple, $\alpha$-recursively enumerable set. This is a consequence of Theorem $1^{\alpha}$, since the ideas of Dekker [1] work quite nicely on regular sets, and since the deficiency set of a function which enumerates a regular set is regular.

3. Metadegrees of $\Pi_{1}^{1}$ sets. Before we prove Theorem 2, we recall some definitions of [7]. The T-predicate of [7] is analogous to that of Kleene [5]. Its definition is:

$$
T_{1}^{1}(B, e, \alpha, \sigma) \leftrightarrow t(e, \sigma)=\langle e, M, N, \alpha, \gamma\rangle \& M \subseteq B \& N \subseteq c B ;
$$

$t(e, \sigma)$ is a special metarecursive function which indexes "deductions". Roughly speaking, $T_{1}^{1}(B, e, \alpha, \sigma)$ says: $B$ is an arbitrary set of recursive ordinals; $e$ is the Gödel number of a finite system of equations $E$; and $t(e, \sigma)$ is the Gödel number of a metafinite "deduction" from $E$ and $B$ whose last line gives the value $\gamma$ of some function at argument $\alpha . U(e, \sigma)$ is a metarecursive function such that if $t(e, \sigma)=\langle e, M, N, \alpha, \gamma\rangle$, then $U(e, \sigma)=\gamma$. Two useful partial functions are:

$$
\begin{aligned}
& \{e\}_{\sigma}^{B}(\alpha)=\gamma \leftrightarrow T_{1}^{1}(B, e, \alpha, \sigma) \& U(e, \sigma)=\gamma \\
& \{e\}^{B}(\alpha)=\gamma \leftrightarrow(E \sigma)\left(\{e\}_{\sigma}^{B}(\alpha)=\gamma\right) \&(\sigma)\left(\{e\}_{\sigma}^{B} \text { is defined } \rightarrow U(e, \sigma)=\gamma\right) .
\end{aligned}
$$

Theorem 2 of [7] states that if $A$ is weakly metarecursive in $B$, then there exists a finite $e$ such that $(\alpha)\left[a(\alpha)=\{e\}^{B}(\alpha)\right]$, where $a(\alpha)$ is the characteristic function of $A$. The converse is also true.

Let $M(e, \sigma)$ and $N(e, \sigma)$ be metarecursive functions such that if $t(e, \sigma)=\langle e, M, N, \alpha, \gamma\rangle$, then $M(e, \sigma)=M$ and $N(e, \sigma)=N$. (We are making use of a standard indexing of the metafinite sets which enables us to speak of partial metarecursive functions that take metafinite sets $(M, N)$ as values.) We introduce two partial functions: 


$$
\begin{aligned}
& { }^{\mathrm{K}}[e]_{\sigma}^{B}(\alpha)=\gamma \leftrightarrow\{e\}_{\sigma}^{B}(\alpha)=\gamma \& K-(N(e, \sigma) \cup B) \text { is infinite; } \\
& { }^{K}[e]^{B}(\alpha)=\gamma \leftrightarrow(E \sigma)\left({ }^{K}[e]_{\sigma}^{B}(\alpha)=\gamma\right) \&(\sigma)\left({ }^{K}[e]_{\sigma}^{B}(\gamma) \text { is defined } \rightarrow U(e, \sigma)=\gamma\right) .
\end{aligned}
$$

The difference between ${ }^{K}[e]^{B}$ and $\{e\}^{B}$ is the key that unlocks the proof of Theorem 2. The "deductions" associated with ${ }^{K}[e]^{B}$ are restricted; each of them permits infinitely many members of $K$ not to be in $B$ or in $N(e, \sigma) \subseteq c B$. The $B$ of Theorem 2 (as well as the $A$ ) have the property that ${ }^{K}[e]^{B} \simeq\{e\}^{B}$ for all $e$ and certain metafinite $K$ 's depending on $e$.

THEOREM 2. There exist two $\Pi_{1}^{1}$ sets of natural numbers such that neither is weakly metarecursive in the other $\left({ }^{2}\right)$.

Proof. We intend to metarecursively enumerate two sets, $A$ and $B$, of natural numbers with an eye to meeting certain requirements. The first set of requirements will insure that $A$ and $B$ are "incomparable". The second set will insure that neither $A$ nor $B$ are metarecursive. The two sets of requirements are redundant, since if $A$ is not weakly metarecursive in $B$, then $A$ is not metarecursive. Sometimes, however, redundancy (overkill) is useful. We will simultaneously attempt to satisfy both sets of requirements, but we will be unable to prove directly that the first set is successfully met. Instead, a direct argument will be given that the second set is met, and then (and only then) it will be possible to show with the aid of the properties of ${ }^{K}[e]^{B}$ that the first set is met.

We handle conflicting requirements by means of a system of priorities similar to that of Friedberg [3]. To be more precise, in the course of the construction, we make only finitely many attempts to satisfy each requirement. Driscoll [2] proves theorems about metarecursively enumerable sets by means of priority arguments in which infinitely many attempts are made to satisfy individual requirements.

We define eight partial metarecursive functions, $A(\sigma), B(\sigma), K(\sigma, e), L(\sigma, e)$, $p(\sigma, e), q(\sigma, e), s(\sigma, e)$ and $v(\sigma, e)$ for all $\sigma$ and all finite $e$ by induction on $\sigma ; p, q, s$ and $v$ will take finite ordinals as values, while the other four functions will take only metafinite sets as values.

Let $r$ be a metarecursive function taking only finite values such that for each $c<\omega$ and $i<4$, the set $\{\sigma \mid r(\sigma)=c \&(E \tau)(\sigma=\tau+j$ and $\tau$ is a limit ordinal $\& j \equiv i(4))\}$ is unbounded.

Stage $\sigma=0$. Let $A(0)=B(0)=\varnothing, K(0, e)=L(0, e)=\{m \mid m<\omega\}, p(0, e)$ $=q(0, e)=2^{2 e+2}$, and $s(0, e)=v(0, e)=2^{2 e+1}$ for all $e$.

Stage $\sigma>0$. If the partial function $\lambda \delta \mid K(\delta, e)$ changes value only finitely often as $\delta$ increases through the ordinals less than $\sigma$, let $K\left(\sigma^{*}, e\right)$ equal its "last" value; i.e.,

(2) It is not hard to show, using Spector [14], that if $B$ is a nonhyperarithmetic, $\Pi_{1}^{1}$ set, then every $\Pi_{1}^{1}$ set is invariantly definable from $B$ (regarded as a set). This last notion is defined in Kreisel [6]. 


$$
(E \delta)_{\delta<\delta}(\rho)\left(\delta \leqq \rho<\sigma \rightarrow K(\rho, e)=K\left(\sigma^{*}, e\right)\right) .
$$

If the partial function changes value infinitely often, let $K\left(\sigma^{*}, e\right)=K(0, e)$. (We will see that the partial function $\lambda \sigma \mid K(\sigma, e)$ changes value only finitely often as $\sigma$ increases through the recursive ordinals.) Define $L\left(\sigma^{*}, e\right), p\left(\sigma^{*}, e\right), q\left(\sigma^{*}, e\right)$, $s\left(\sigma^{*}, e\right)$ and $v\left(\sigma^{*}, e\right)$ similarly.

Let $c=r(\sigma)$ and $\sigma=\tau+j$, where $\tau$ is a limit ordinal and $j$ is finite. Let $A\left(\sigma^{*}\right)=\bigcup\{A(\delta) \mid \delta<\sigma\}$ and $B\left(\sigma^{*}\right)=\bigcup\{B(\delta) \mid \delta<\sigma\}$.

Case 1. $j \equiv 0$ (4).

Case 1.1. $s\left(\sigma^{*}, c\right) \notin A\left(\sigma^{*}\right)$ and there is a $\gamma<\sigma$ such that $\{c\}_{\gamma}^{\phi}\left(s\left(\sigma^{*}, c\right)\right)=1$. Define $A(\sigma)=A\left(\sigma^{*}\right) \cup\left\{s\left(\sigma^{*}, c\right)\right\}$. Set $B(\sigma)=B\left(\sigma^{*}\right), K(\sigma, e)=K\left(\sigma^{*}, e\right), L(\sigma, e)$ $=L\left(\sigma^{*}, e\right), q(\sigma, e)=q\left(\sigma^{*}, e\right)$ and $s(\sigma, e)=s\left(\sigma^{*}, e\right)$. If $L(\sigma, e)-B(\sigma)$ is finite for some $e$, then define $p(\sigma, e)=p\left(\sigma^{*}, e\right)$ and $v(\sigma, e)=v\left(\sigma^{*}, e\right)$ for all $e$. Suppose $L(\sigma, e)-B(\sigma)$ is infinite for all $e$; then set:

$$
\begin{aligned}
& p(\sigma, e)=p\left(\sigma^{*}, e\right) \text { if } e<c ; \\
& v(\sigma, e)=v\left(\sigma^{*}, e\right) \text { if } e \leqq c ; \\
& p(\sigma, k)=\mu x[x \in L(\sigma, k)-B(\sigma) \&(E y)(y \in L(\sigma, k)-B(\sigma) \\
& \left.\left.\qquad \& x>y>v(\sigma, k)+v\left(\sigma^{*}, k\right)+p\left(\sigma^{*}, k\right)\right)\right] \text { if } k \geqq c ; \\
& v(\sigma, k)=\mu x[x \in L(\sigma, k)-B(\sigma) \& x>p(\sigma, k-1)] \text { if } k>c .
\end{aligned}
$$

Case 1.2. $s\left(\sigma^{*}, c\right)$ does not satisfy the hypothesis of case 1.1. Change nothing and proceed to stage $\sigma+1$.

Case 2. $j \equiv 1$ (4).

Case 2.1. $p\left(\sigma^{*}, c\right) \notin B\left(\sigma^{*}\right)$ and there is a $\gamma<\sigma$ such that ${ }^{K\left(\sigma^{*}, c\right)}[c]_{\gamma}^{A\left(\sigma^{*}\right)}\left(p\left(\sigma^{*}, c\right)\right)=1$; let $\delta$ be the least such $\gamma$. Define $B(\sigma)=B\left(\sigma^{*}\right) \cup\left\{p\left(\sigma^{*}, c\right)\right\}$. Set $A(\sigma)=A\left(\sigma^{*}\right)$. Let $L(\sigma, e)=L\left(\sigma^{*}, e\right), p(\sigma, e)=p\left(\sigma^{*}, e\right)$ and $v(\sigma, e)=v\left(\sigma^{*}, e\right)$. Define $K(\sigma, e)=K\left(\sigma^{*}, e\right)$ for all $e<c, K(\sigma, c)=K\left(\sigma^{*}, c\right)-N(c, \delta)$, and $K(\sigma, e)=$ $K(\sigma, c)$ for all $e>c$. If $K(\sigma, e)-A(\sigma)$ is finite for some $e$, then define $q(\sigma, e)=q\left(\sigma^{*}, e\right)$ and $s(\sigma, e)=s\left(\sigma^{*}, e\right)$ for all $e$. Suppose $K(\sigma, e)-A(\sigma)$ is infinite for all $e$; then set:

$$
\begin{aligned}
q(\sigma, e) & =q\left(\sigma^{*}, e\right) \text { if } e<c ; \\
s(\sigma, e) & =s\left(\sigma^{*}, e\right) \text { if } e \leqq c ; \\
q(\sigma, k) & =\mu x[x \in K(\sigma, k)-A(\sigma) \&(E y)(y \in K(\sigma, k)-A(\sigma) \\
& \left.\left.\quad \& x>y>s(\sigma, k)+s\left(\sigma^{*}, k\right)+q\left(\sigma^{*}, k\right)\right)\right] \text { if } k \geqq c ; \\
s(\sigma, k) & =\mu x[x \in K(\sigma, k)-A(\sigma) \& x>q(\sigma, k-1)] \text { if } k>c .
\end{aligned}
$$

Case 2.2. $p\left(\sigma^{*}, c\right)$ does not satisfy the hypothesis of Case 2.1. Change nothing and proceed directly to stage $\sigma+1$. 
Case 3. $j \equiv 2$ (4). Similar to case 1 with $A$ and $B$ interchanged; we also interchange $s$ and $v, K$ and $L$, and $p$ and $q$.

Case 4. $j \equiv 3$ (4). Similar to Case 2 with $A$ and $B$ interchanged. If case 4.1 holds and $L(\sigma, e)-B(\sigma)$ is infinite for all $e$, then set:

$$
\begin{aligned}
& p(\sigma, e)=p\left(\sigma^{*}, e\right) \text { if } e \leqq c ; \\
& v(\sigma, e)=v\left(\sigma^{*}, e\right) \text { if } e \leqq c+1 \text {; } \\
& p(\sigma, k)=\mu x[x \in L(\sigma, k)-B(\sigma) \&(E y)(y \in L(\sigma, k)-B(\sigma) \\
& \left.\left.\& x>y>v(\sigma, k)+v\left(\sigma^{*}, k\right)+p\left(\sigma^{*}, k\right)\right)\right] \text { if } k>c ; \\
& v(\sigma, k)=\mu x[x \in L(\sigma, k)-B(\sigma) \& x>p(\sigma, k-1)] \text { if } k>c+1 \text {. }
\end{aligned}
$$

That completes the construction. Note that we are using the fact that the predicate, $\alpha$ is the index of an infinite, metafinite set, is metarecursive.

The system of priorities we are following is buried in the above equations; we make it explicit. Our requirements are: $B \neq\{e\}^{A}, A \neq\{e\}^{B}, B \neq\{e\}^{\phi}$, and $A \neq\{e\}^{\phi}$, where $e \geqq 0 . B \neq\{c\}^{A}$ has higher priority then $A \neq\{e\}^{B}$ for all $e \geqq c$. (Any conflict between two requirements is resolved in favor of the requirement of higher priority.) $A \neq\{c\}^{B}$ has higher priority than $A \neq\{e\}^{\phi}, B \neq\{e\}^{\phi}$, and $B \neq\{e\}^{A}$ for all $e>c . A \neq\{c\}^{\phi}$ and $B \neq\{c\}^{\Phi}$ have higher priority than $B \neq\{e\}^{A}$ for all $e \geqq c$.

Let $f(\sigma)$ be a function from the recursive ordinals into the recursive ordinals. We say $f(\sigma)$ changes value only finitely often as $\sigma$ increases through the recursive ordinals if there exists a finite sequence, $0=\sigma_{0}<\sigma_{1}<\cdots<\sigma_{m}=\omega_{1}$, such that for each $i<m, f(\sigma)$ is constant when restricted to $\sigma_{i} \leqq \sigma<\sigma_{i+1}$.

LEMMA 1. Let $f(\sigma, e)$ denote any one of the following six functions: $K(\sigma, e)$, $L(\sigma, e), p(\sigma, e), q(\sigma, e), s(\sigma, e)$ or $v(\sigma, e)$. Then for each $e$, the function $\lambda \sigma \mid f(\sigma, e)$ changes value only finitely often as $\sigma$ increases through the recursive ordinals.

Proof. First we show $\lambda \sigma \mid p(\sigma, e)$ and $\lambda \sigma \mid v(\sigma, e)$ change value only finitely often for each $e$ by an infinite descent on $e$. Fix $e$ and suppose otherwise. Then Case 1.1 holds for infinitely many $\sigma$ with $c \leqq e$ or Case 4.1 holds for infinitely $\sigma$ with $c+1 \leqq e$. Then $\lambda \sigma \mid s\left(\sigma, c^{\prime}\right)$ changes value infinitely often for some $c^{\prime} \leqq e$ or $\lambda \sigma \mid q\left(\sigma, c^{\prime}\right)$ changes value infinitely often for some $c^{\prime}<e$. Each of these last two alternatives implies that Case 2.1 or Case 3.1 holds for infinitely many $\sigma$ and some $c<e$. If Case 2.1 holds for infinitely many $\sigma$ and $c=c^{\prime \prime}<e$, then $\lambda \sigma \mid p\left(\sigma, c^{\prime \prime}\right)$ changes value infinitely often. If Case 3.1 holds for infinitely many $\sigma$ and $c=c^{\prime \prime}<e$, then $\lambda \sigma \mid v\left(\sigma, c^{\prime \prime}\right)$ changes value infinitely often.

Infinitely many changes in $\lambda \sigma \mid s(\sigma, e)$ imply infinitely many changes in $\lambda \sigma \mid p(\sigma, c)$ for some $c<e$ or infinitely many changes in $\lambda \sigma \mid v(\sigma, c)$ for some $c<e$. A similar relationship holds between $q$ and $p$ and $v$. Infinitely many changes in $\lambda \sigma \mid K(\sigma, e)$ 
imply infinitely many changes in $\lambda \sigma \mid p(\sigma, c)$ for some $c<e$ by Case 2.1. A similar relationship holds between $L$ and $q$.

LEMMA 2. For each $e$ and $\sigma, K(\sigma, e)-A(\sigma)$ and $L(\sigma, e)-B(\sigma)$ are infinite.

Proof. Fix $e \geqq 0$ and $\sigma>0$. First we show $L\left(\sigma^{*}, e\right)-B\left(\sigma^{*}\right)$ is infinite. By Lemma 1 , there is a least $\gamma<\sigma$ such that $L\left(\sigma^{*}, e\right)=L(\sigma, e)=L(\gamma, e)=L(\tau, e)$ whenever $\sigma>\tau>\gamma$. Fix $m$; we locate a member of $L(\gamma, e)-B\left(\sigma^{*}\right)$ greater than $m$. Clearly, $f<v\left(\sigma^{*}, f\right)<p\left(\sigma^{*}, f\right)<v\left(\sigma^{*}, f+1\right)$ for all $f$. Let $f$ be such that $e+m<f$. Then there is a $y$ such that $m<v\left(\sigma^{*}, f\right)<y<p\left(\sigma^{*}, f\right)$ and $y \in L(\gamma, e)-B\left(\sigma^{*}\right)$. We prove this last assertion. Let $\tau$ be the least $\alpha$ such that $\alpha<\sigma$ and $p(\alpha, f)=p\left(\sigma^{*}, f\right)=p(\delta, f)$ when $\sigma>\delta>\alpha$; then $p(\tau, d)=p\left(\sigma^{*}, d\right)$ $=p(\delta, d)$ when $\sigma>\delta>\tau$ and $d \leqq f$. Then $y \in L(\tau, f)-B(\tau)$, and so $y \in L(\tau, e)-B(\tau)$, since $e<f$. We claim $\gamma \leqq \tau$; if $\tau<\gamma$, then $L(\gamma, e) \neq L\left(\gamma^{*}, e\right)$ (by definition of $\gamma$ ) and $p(\gamma, f) \neq p\left(\gamma^{*}, f\right)$ (by Case 4.1); but $p(\gamma, f)=p\left(\gamma^{*}, f\right)$ if $\tau \leqq \gamma$. Since $\gamma \leqq \tau$, we have $y \in L(\gamma, f)-B(\tau)$. We still must see that $y \notin B\left(\sigma^{*}\right)-B(\tau)$. Suppose $y \in B(\delta)-B\left(\delta^{*}\right)$ where $\sigma>\delta>\tau$. Then $y=p\left(\delta^{*}, c\right)$ or $y=v\left(\delta^{*}, c\right)$. But $y \neq p\left(\delta^{*}, c\right)$, because $p\left(\delta^{*}, f-1\right)<v\left(\delta^{*}, f\right)<y<p\left(\delta^{*}, f\right)$; similarly, $y \neq v\left(\delta^{*}, c\right)$. Finally, $y \in L(\gamma, e)-B\left(\delta^{*}\right)$, since $e<f$.

Now we show $L(\sigma, e)-B\left(\sigma^{*}\right)$ is infinite. If $L(\sigma, e) \neq L\left(\sigma^{*}, e\right)$, Case 4.1 holds. It follows that ${ }^{L\left(\sigma^{*}, c\right)}[c]_{\delta}^{B\left(\sigma^{*}\right)}\left(q\left(\sigma^{*}, c\right)\right)=1$, and that $L(\sigma, c)=L\left(\sigma^{*}, c\right)-N(c, \delta)$. From (12) we obtain $L\left(\sigma^{*}, c\right)-\left(N(c, \delta) \cup B\left(\sigma^{*}\right)\right)$ is infinite. Since $e \geqq c$, we have $L(\sigma, e)=L(\sigma, c)$ and $L(\sigma, e)-B(\sigma)$ is infinite. A similar argument shows $K(\sigma, c)-A(\sigma)$ is infinite.

Lemma 3. For each $c, A \neq\{c\}^{\phi}$ and $B \neq\{c\}^{\phi}$.

Proof. Fix $c$ and let $s(c)=\lim _{\sigma} s(\sigma, c)$ (the final value of $s(\sigma, c)$ ). Suppose $\{c\}^{\phi}(s(c))$ is defined. Let $\gamma$ be the least $\delta$ such that $s(c)=s(\delta, c)=s(\sigma, c)$ for all $\sigma>\delta$. Then $s(c) \notin A(\gamma)$ by Lemma 2. If there is a $\sigma$ such that $\{c\}_{\sigma}^{\phi}(s(c))=1$, then $s(c) \in A$ by Case 1 . If $\{c\}^{\phi}(s(c))=0$, then $s(c) \notin A$, since $s$ and $q$ have disjoint ranges. A similar argument shows $B \neq\{c\}^{\phi}$.

Lemma 4. For each $c, B \neq\{c\}^{A}$ and $A \neq\{c\}^{B}$.

Proof. Fix $c$ and let $p(c)=\lim _{\sigma} p(\sigma, c)$. Suppose $\{c\}^{A}(p(c))$ is defined. Let $\rho$ be the least $\delta$ such that $p(e)=p(\delta, e)=p(\sigma, e)$ for all $\sigma>\delta$ and $e \leqq c$. Then $p(c) \notin L(\rho, c)-B(\rho)$ by Lemma 2. Suppose $p(c) \in B$. Then Case 2.1 must hold at stage $\sigma>\rho$ and ${ }^{K\left(\sigma^{*}, c\right)}[c]_{\delta}^{A\left(\sigma^{*}\right)}(p(c))=1$. It is enough to show $N(c, \delta) \cap A=\varnothing$, since $\{c\}^{A}(p(c))$ is defined. Now $K(\sigma, e) \cap N(c, \delta)=\varnothing$ for all $e \geqq c$. But $K(\sigma, c)=K(\alpha, c)$ for all $\alpha>\sigma$, since $\sigma>\rho$; consequently, $K(\tau, e) \subseteq K(\sigma, c)$ for all $\tau \geqq \sigma$ and $e \geqq c$. Thus $N(c, \delta) \cap K(\tau, e)=\varnothing$ for all $\tau \geqq \sigma$ and $e \geqq c$. Then $q(\tau, e)$ and $s(\tau, e) \notin N(c, \delta)$ for all $\tau \geqq \sigma$ and $e \geqq c$. So if $N(c, \delta) \cap A \neq \varnothing$, there must be an $f<c$ and a $\tau>\sigma$ such that $q\left(\tau^{*}, f\right) \in N(c, \delta) \cap A(\tau)$ or 
$s\left(\tau^{*}, f\right) \in N(c, \delta) \cap A(\tau)$. Both of these alternatives are ruled out by the fact that $\sigma>\rho$.

Now suppose $p(c) \notin B$. We claim $\{c\}^{A}(p(c)) \neq 1$; suppose otherwise. Then there is a $\gamma$ and a $\beta$ such that $\gamma<\beta$ and $\{c\}_{\gamma}^{A\left(\sigma^{*}\right)}(p(c))=1$ for all $\sigma \geqq \beta$; in addition, $M(c, \gamma) \subseteq A$ and $N(c, \gamma) \subseteq c A$. Assume $\beta \geqq \rho$. Then $K(\sigma, c)=K(\beta, c)$ for all $\sigma \geqq \beta$. Any element added to $A$ after stage $\beta$ must be a member of $K(\beta, c)$ with finitely many exceptions (at most $c$ ). Consider $K(\beta, c)-(N(c, \gamma) \cup A(\sigma)$ ) for any $\sigma \geqq \beta$; it must be infinite, since otherwise $(A-A(\beta))-(N(c, \gamma) \cup A(\sigma))$ would be finite and $A$ would be metafinite. But Lemma 4 says $A$ is not metafinite. But then ${ }^{K\left(\sigma^{*}, c\right)}[c]_{\gamma}^{A\left(\sigma^{*}\right)}(p(c))=1$ for all $\sigma \geqq \beta$, and some instance of case 2.1 would put $p(c)$ in $B$. A similar argument shows $A \neq\{c\}^{B}$.

Note that it was not sufficient for us to know that various $\operatorname{limits}_{\text {such }} \lim _{\sigma} p(\sigma, e)$ and $\lim _{\sigma} L(\sigma, e)$ existed. Our key lemma, Lemma 2, used Lemma 1 very heavily; we had to know that the function $\lambda_{\sigma} \mid L(\sigma, e)$ changed value only finitely often as $\sigma$ increased through the recursive ordinals. This mode of argument seems to generalize only to those admissible ordinals which project into cardinals. We make a conjecture on this matter in $\$ 7$.

THEOREM 3. Every countable partial ordering is embeddable in the partial ordering of metadegrees of $\Pi_{1}^{1}$ sets.

Proof. Combine the argument of Theorem 2 of $\$ 5$ of [11] with the proof of Theorem 2 above. The proof of Lemma 2 above becomes more complicated, but no conceptual change is required.

4. Subgeneric sets. The notion of subgeneric set is due to Kreisel. Before we give the definition, we recall some features of Kripke's equation calculus introduced in [8] and sketched in [7]. Kripke's calculus is modeled on Kleene's [5]. The terms include function letters and numerals denoting recursive ordinals. The deduction rules are similar to the substitution rules of Kleene with one exception, rule (R3). By means of (R3) it is possible to deduce $(x)_{x<\beta}(f(x)=0)$ from the set of premises $\{f(\alpha)=0 \mid \alpha<\beta\}$, where $\alpha$ and $\boldsymbol{\beta}$ are numerals denoting the recursive ordinals $\alpha$ and $\beta$. (Note: in Kripke's formalism, the formula $(x)_{x<\beta}(j(x)=0)$ is rendered as the equation $(E x<\beta)(f(x)=1$.) Kripke's calculus can be thought of as the usual means of determining the truth-value of formulas concerning function-values in which all quantifiers are bounded. Let $E$ be a finite set of equations. Let $S_{0}^{E}=E$, and for each ordinal $\alpha$ (not necessarily recursive), let $S_{\alpha}^{E}$ be the set of all equations immediately deducible from $\bigcup\left\{S_{\gamma}^{E} \mid \gamma<\alpha\right\}$. Each member of $S_{\alpha}^{E}$ is an immediate consequence of finitely many premises in $\bigcup\left\{S_{\gamma}^{E} \mid \gamma<\alpha\right\}$ save those which result from (R3). If (R3) is applied to the members of $\bigcup\left\{S_{\gamma}^{E} \mid \gamma<\alpha\right\}$, then we require that the $\beta$ mentioned in each use of (R3) be less than $\alpha$; thus every member of $S_{\omega}^{E}$ is an immediate consequence of finitely many members of $\bigcup\left\{S_{m}^{E} \mid m<\omega\right\}$. Kripke shows $S_{\omega_{1}}^{E}=$ $\bigcup\left\{S_{\gamma}^{E} \mid \gamma<\omega_{1}\right\}$, where $\omega_{1}$ is the least nonrecursive ordinal. 
In [7] it was observed that a function $\Phi$ is metarecursive if and only if for some $E$ with principal function letter $f, \Phi(\gamma)=\delta \leftrightarrow f(\gamma)=\delta \in S_{\omega_{1}}^{E}$. We now characterize the relation, "weakly metarecursive in", in a similar fashion. Let $B$ be an arbitrary set of recursive ordinals. Let $S_{0}^{E, g}=E \cup\{g(\alpha)=\mathbf{1} \mid \alpha \notin \beta\} \vee\{g(\alpha)=\mathbf{0} \mid \alpha \in \beta\}$; for each $\alpha>0$, let $S_{\alpha}^{E, g}$ equal the set of all immediate consequences of $\bigcup\left\{S_{\gamma}^{E, g} \mid \gamma<\alpha\right\}$. (Do not forget the condition made in the definition of $S_{\gamma}^{E}$ concerning the use of (R3).) It is not true that $S_{\omega_{1}}^{E, g}=\bigcup\left\{S_{\gamma}^{E, g} \mid \alpha<\omega_{1}\right\}$ for all $B$ and $E$. It is possible to make deductions whose heights are not recursive ordinals. It is even possible to make deductions of recursive ordinal height which are not metafinite; i.e., for some $B$ and recursive $\alpha, S_{\gamma}^{E, g}$ may contain an equation deducible from $B$ whose deduction-tree is not a metafinite object. Let

$$
S^{E, g}=\bigcup\left\{S_{\gamma}^{E, g} \mid \alpha<\text { aleph-one }\right\}
$$

We define $A \leqq_{m c} B$ ( $A$ is metafinitely computable from $B$ ) to mean that for some finite $E$ containing the principal function letter $f$ and the given function letter $g$,

$$
\begin{aligned}
& \alpha \notin A \leftrightarrow f(\alpha)=\mathbf{0} \in S^{E, g, m} \\
& \alpha \notin A \leftrightarrow f(\alpha)=\mathbf{1} \in S^{E, g, m},
\end{aligned}
$$

where $S^{E, g, m}$ is the set of all members of $S^{E, g}$ whose deduction-trees are metafinite. Clearly, the height of each member of $S^{E, g, m}$ is a recursive ordinal. $A \leqq_{m c} B$ means the membership relation of $A$ is computable from $B$ using the equation calculus and metafinite deductions only.

THEOREM 4. $A \leqq_{w} B \leftrightarrow A \leqq_{m c} B$.

Proof. If $A \leqq_{w} B$, then the computations used to decide $A$ from $B$ are metafinite and are metarecursively enumerable; consequently, $A \leqq_{m c} B$. Suppose $A \leqq_{m c} B$ by $E$. Each deduction permitted is a metafinite object; the roots of each deduction of $\alpha \in A$ or $\alpha \notin A$ consist of a metafinite set of membership conditions satisfied by $B$. The set of all metafinite deductions is metarecursively enumerable. It is not difficult to define the partial metarecursive functions needed to describe how each decision about the membership of $A$ is determined by a metafinite neighborhood condition satisfied by $B$. Then $A \leqq_{w} B$.

We say $B$ is subgeneric if for every $E$, we have $S_{\omega_{1}}^{E, g}=\bigcup\left\{S_{\gamma}^{E, g} \mid \alpha<\omega_{1}\right\}$, where $S_{0}^{E, g}$ includes the diagram of $B$. Intuitively, $B$ is subgeneric if every equation deducible from $E$ and $B$ is deducible from $E$ and $B$ at some recursive ordinal. It is easily checked that every metarecursive set is subgeneric, and that every bounded, nonmetarecursive, metarecursively enumerable set is not subgeneric (first observed by Kreisel). In a letter to the author, Kreisel asked: do there exist subgeneric, nonmetarecursive, metarecursively enumerable sets? The reason 
for his interest lies in the light shed by the notion of subgenericity on the various notions of reducibility discussed in [6]. It turns out that one can prove the existence of nonmetarecursive, subgeneric sets by a standard measure-theoretic argument or by a "forcing" argument. (In fact, with probability one, a randomly chosen set of recursive ordinals is subgeneric.) However, a much stronger result, expressed by Theorem 6, can be obtained by the priority method. First we introduce a generally stronger notion. We say $B$ is hyperregular if the range of each function weakly metarecursively in $B$, when restricted to a metafinite set, is bounded by a recursive ordinal. We will see that a set is both regular and hyperregular if and only if all possible deductions from the set are metafinite.

THEOREM 5. A regular set of recursive ordinals is hyperregular if and only if it is subgeneric.

Proof. Suppose $B$ is regular and subgeneric. First we show $B$ is completely regular: every bounded set weakly metarecursive in $B$ is metafinite. Let $C$ be bounded and weakly metarecursive in $B$. Let $\gamma$ be the least upper bound of $C$. There exist functions $m(\delta)$ and $n(\delta)$ weakly metarecursive in $B$ such that the truthvalue of $\delta \in C$ is deducible from $B$ (and some $E$ not depending on $\delta$ ) using only membership information about $B$ expressed by $K_{m(\delta)} \subseteq B$ and $K_{n(\delta)} \subseteq c B$. Suppose $\rho=\{m(\delta)+n(\delta) \mid \delta<\gamma\}$ is unbounded. Then $Q$, a $\Pi_{1}^{1}$ set of unique notations, is computable from $\{|\tau| \mid \tau \in p\}$ by means of the unrestricted equation calculus. But then $Q$ is computable from $B$, and consequently, $Q$ is subgeneric. No nonmetarecursive $\Pi_{1}^{1}$ set is subgeneric, so $\rho$ must be bounded. It follows from the regularity of $B$ that $C$ is metafinite.

Let $f$ be weakly metarecursive in $B$, and let $K$ be a metafinite set. Then $\{\langle|\alpha|,|f(\alpha)|\rangle \mid \alpha \in K\}$ is bounded and weakly metarecursive in $B$; by the previous paragraph, it must be metafinite.

Now suppose $B$ is regular and hyperregular. Let $E$ be a finite set of equations, and let $\beta$ be a recursive ordinal. Suppose each member of $\{f(\gamma)=0 \mid \gamma<\beta\}$ is deducible from $E$ and $B$ by means of a metafinite deduction. Then there exist functions $s(\gamma)$ and $t(\gamma)$ weakly metarecursive in $B$ such that some metafinite deduction of $f(\gamma)=0$ from $E$ and $B$ uses only the membership information about $B$ expressed by $K_{s(\gamma)} \subseteq B$ and $K_{t(\gamma)} \subseteq c B$. But $\{s(\gamma) \mid \gamma<\beta\}$ and $\{t(\gamma) \mid \gamma<\beta\}$ are bounded. It follows from the regularity of $B$ that all members of $\{f(\gamma)=0 \mid \gamma<\beta\}$ are deducible from $E$ and a fixed metafinite set of membership facts about $B$.

COROllary 1. A metarecursively enumerable set is subgeneric if and only if it is regular and hyperregular.

Proof. Any subgeneric metarecursively enumerable set is regular.

THEOREM 6. There exists a nonmetarcursive, metarecursively enumerable, regular, hyperregular set of recursive ordinals. 
Proof. Let $R_{0}, R_{1}, \cdots, R_{e}, \cdots(e<\omega)$ be the standard enumeration of the metarecursively enumerable sets; let $R_{e}^{\sigma}$ be all the members of $R_{e}$ enumerated prior to stage $\sigma ; \lambda \sigma e \mid R_{e}^{\sigma}$ is a metarecursive function which takes metafinite sets as values. Let $r(\sigma)$ be a metarecursive function taking only finite values such that for each $c$, the set $\{\sigma \mid r(\sigma)=c\}$ is unbounded. At stage $\sigma$ we consider putting an element into $A$ in order to insure that $A \cap R_{e} \neq \varnothing$ if $R_{e}$ is unbounded; in this fashion we make $A$ nonrecursive. At the same time we try to preserve all values of $\{e\}^{A}(m)(m<\omega)$ as they develop. We do not add an element of $R_{c}$ to $A$ if it would change the apparent value of $\{e\}^{A}(m)$ for any $m<\omega$ and $e<c$. This system of priorities guarantees that any set of natural numbers metarecursive in $A$ will be metarecursive, since for each $e,\{e\}^{A}$ will be "injured" only finitely often. It will follow that $A$ is regular and hyper-regular.

Stage $\sigma \geqq 0$. Let $A\left(\sigma^{*}\right)=\bigcup\{A(\delta) \mid \delta<\sigma\}$. For each $e<\omega$, if for all $m<\omega$ there is a $\delta<\omega$ such that $\{e\}_{\delta}^{A\left(\sigma^{*}\right)}(m)$ is defined, let $\gamma(e, \sigma)$ be the least $\gamma$ such that for all $m<\omega$, there is a $\delta<\sigma$ such that $\{e\}_{\delta}^{A\left(\sigma^{*}\right)}(m)$ is defined and $\gamma>$ all members of $N(e, \delta)$; otherwise, let $\gamma(e, \sigma)=0$. We define

$$
d(e, \sigma)=\mu x(x \geqq \gamma(e, \sigma) \&(\rho)(\rho<\sigma \rightarrow x \geqq d(e, \rho)))
$$

for each $e$. Let $r(\sigma)=c$. If $R_{c}^{\sigma} \cap A\left(\sigma^{*}\right)=\varnothing$, and if there is a $\rho$ such that $\rho \in R_{c}^{\sigma} \&(e)_{e<c}(\rho>d(e, \sigma)) \& \rho$ greater than any member of

$$
\bigcup\left\{R_{r(\delta)}^{\delta} \cap\left(A(\delta)-A\left(\delta^{*}\right)\right) \mid \delta<\sigma \& r(\delta)<c\right\}
$$

by at least $\omega$, add the least such $\rho$ to $A\left(\sigma^{*}\right)$ to obtain $A(\sigma)$; otherwise, let $A(\sigma)=$ $A\left(\sigma^{*}\right)$.

Let $A=\bigcup\left\{A(\sigma) \mid \sigma<\omega_{1}\right\}$.

LEMMA 8. For each $e, \lim _{\sigma} d(e, \sigma)$ exists.

Proof. Fix $e$. For each $c \leqq e$, there is at most one $\sigma$ such that at stage $\sigma$ : $A$ is augmented for the sake of $R_{c}$. (This is the purpose of the clause $R_{c}^{\sigma} \cap A\left(\sigma^{*}\right)=\varnothing$.) Let $\sigma$ be so large that $A$ is not augmented at stage $\rho$ for the sake of $R_{c}$ for any $\rho \geqq \sigma$ and $c \leqq e$. The set $\{\gamma(e, \rho) \mid \rho \geqq \sigma\}$ must have a maximum value, since if $\gamma(e, \delta)>0$ for some $\delta \geqq \sigma$, then $\gamma(e, \rho) \leqq \gamma(e, \delta)$ for all $\rho>\delta$; suppose $\tau \geqq \sigma$ is such that $\gamma(e, \tau)$ is the maximum value. Then $d(e, \rho)=d(e, \tau)$ for all $\rho \geqq \tau$. (Note that if $\alpha$ is added to $A$ for the sake of $R_{c}$ at stage $\rho \geqq \sigma$, then $c>e$ and $\alpha>d(e, \rho)$.)

LEMMA 9. A is not metarecursive.

Proof. First we show $c A$ is unbounded. Fix $\alpha$; we claim $\{\beta \mid \alpha<\beta<\alpha+\omega\} \cap A$ is finite. Let $\tau$ and $\sigma$ be such that $\alpha<\tau<\alpha+\omega, \tau \in A(\sigma)$, and $A\left(\sigma^{*}\right) \cap\{\beta \mid \alpha<\beta<\alpha+\omega\}=\varnothing$. Let $r(\sigma)=c$. If $\rho$ is added to $A$ at stage $\delta>\sigma$ 
for the sake of $R_{d}$, where $d>c$, then $\rho \geqq \alpha+\omega$, since $\rho$ must be greater than $\tau$ by at least $\omega$. Thus any member of $\{\beta \mid \alpha<\alpha+\omega\}$ added to $A$ after stage $\sigma$ must be added for the sake of $R_{d}$, where $d<c$; consequently, $\{\beta \mid \alpha<\beta<\alpha+\omega\}$ $\cap A$ has at most $c+1$ members.

Fix $c$ and suppose $R_{c}$ is unbounded. Let $\rho$ have the following properties: $\rho \in R_{c},(e)_{e<c}\left(\rho>\lim _{\sigma} d(e, \sigma)\right)$, and $\rho$ is greater by at least $\omega$ than any member of $\bigcup\left\{R_{r(\delta)}^{\delta} \cap\left(A(\delta)-A\left(\delta^{*}\right)\right) \mid \delta<\omega_{1} \& r(\delta)<c\right\}$; such a $\rho$ exists by Lemma 8 and the fact that for each $e$, there is at most one $\delta$ such that $R_{\sigma(\delta)}^{\delta} \cap\left(A(\delta)-A\left(\delta^{*}\right)\right) \neq \varnothing \& r(\delta)=e$. Then $A \cap R_{c}$ contains a member less than or equal to $\rho$.

LEMma 10. For each $e$, if $\{e\}^{A}(m)$ is defined for all $m<\omega$, then $\{e\}^{A}$ agrees with some metarecursive function on the natural numbers.

Proof. Fix $e$ and suppose $\{e\}^{A}(m)$ is defined for all $m<\omega$. Fix $m$; we show how to compute $\{e\}^{A}(m)$ metaeffectively. Let $\sigma$ be so large that $A$ is not augmented at stage $\rho$ for the sake of $R_{c}$ for any $\rho \geqq \sigma$ and $c \leqq e$, and such that $d(e, \rho)=d(e, \sigma)$ for all $\rho \geqq \sigma$. Let $\tau(m) \geqq \sigma$ have the property that for some $\delta<\tau(m),\{e\}_{\sigma}^{A\left(\tau(m)^{*}\right)}(m)$ is defined; $\tau(m)$, as a function of $m$, is weakly metarecursive. Then $\{e\}_{\rho}^{A\left(\tau(m)^{*}\right)}(m)$ is defined and $d(e, \tau(m))>$ all members of $N(e, \rho)$. But then $d(e, \gamma)>$ all members of $N(e, \rho)$ for all $\gamma \geqq \tau(m)$, and so $N(e, \rho) \subseteq c A$; consequently, $\{e\}_{\rho}^{A\left(\tau(m)^{*}\right)}(m)=\{e\}^{A}(m)$.

It follows from Lemma 10 that $A$ is both regular and hyperregular.

If $A$ and $B$ are sets of recursive ordinals, let $A \leqq_{c} B$ mean that $A$ is computable from $B$ by means of Kripke's equation calculus; of course, deductions whose heights are nonrecursive ordinals are permitted. If $B$ is regular and hyperregular, and $A$ is computable from $B$, then $A$ is weakly metarecursive in $B$.

Kreisel considers several notions of invariant definability in [6] and in private communications. In a future publication he will present the most appealing of these notions; for now, let us denote it by $\varliminf_{I}$, and let us be content to hear some of Kreisel's observations concerning it; $\leqq_{I}$ is transitive; if $A$ and $B$ are sets of recursive ordinals and $A \leqq_{I} B$, then $A \leqq_{c} B$; if $A$ and $B$ are sets of natural numbers, then $A \leqq_{I} B \leftrightarrow A \leqq_{c} B \leftrightarrow A$ is hyperarithmetic in $B$.

It follows from Theorem 7 that there exist two metarecursively enumerable sets such that neither is invariantly definable from the other.

THEOREM 7. There exist two metarecursively enumerable sets such that neither is computable from the other by means of Kripke's equation calculus.

Proof. We lift Theorem 1 of $\S 5$ of [11] up into metarecursion theory as follows: each regular, nonmetarecursively enumerable set $B$ is the disjoint union of two metarecursively enumerable sets of incomparable metadegrees. The regularity of $B$ is all-important; we need to know that for each $\alpha$ we eventually finish enu- 
merating all members of $B$ less than $\alpha$. Except for this point, the lifting up is routine. Let $B$ be regular and hyperregular. Then $B=B_{0} \cap B_{I}, B_{0} \cap B_{1}=\Phi$, and the metadegrees of $B_{0}$ and $B_{1}$ are incomparable. It follows that $B_{0}$ and $B_{1}$ are regular and hyperregular; but then neither is computable from the other.

We conclude the present section by outlining some generalizations to arbitrary admissible ordinals. At the beginning of this section we sketched Kripke's equation calculus for $\omega_{1}$. The only change needed for an arbitrary admissible $\alpha$ is the introduction of numerals forordinals less than $\alpha$. We define $S_{\gamma}^{E}$ and $S_{\gamma}^{E, g}$ as before. By the definition of admissibility, we have $S_{\alpha}^{E}=\bigcup\left\{S_{0}^{E} \mid \gamma<\alpha\right\}$. Let $B$ be a subset of $\alpha$. We say $B$ is subgeneric if for every $E, S_{\alpha}^{E, g}=\bigcup\left\{S_{\gamma}^{E, g} \mid \gamma<\alpha\right\}$, where $S_{0}^{E g}$ includes the diagram of $B$. We say $B$ is hyperregular if the range of each function weakly $\alpha$-recursive in $B$, when restricted to a set bounded by an ordinal less than $\alpha$, is bounded by an ordinal less than $\alpha$.

THEOREM 8. The following three properties of a regular set $B$ are equivalent to hyperregularity:

(a) every deduction from $B$ is $\alpha$-finite;

(b) the range of each function weakly $\alpha$-recursive in $B$, when restricted to an $\alpha$-finite set, is $\alpha$-finite;

(c) the restriction of each function weakly $\alpha$-recursive in $B$ to an $\alpha$-finite set is an $\alpha$-finite partial function.

Proof. We indicate only that hyperregularity plus regularity implies property (a). The second half of the proof of Theorem 5 uses no special properties of recursive ordinals, generalizes to an arbitrary admissible $\alpha$ with no change, and gives the desired result.

COROLlARY 2. Each regular, hyperregular set is subgeneric.

THEOREM 9. For each admissible $\alpha$, there exists a non- $\alpha$-recursive, a-recursively enumerable, regular, hyperregular set.

Proof. First we mimic the proof of Theorem 6; the argument splits into two cases distinguished by the truth-value of the statement: $\alpha^{*}$, the projectum of $\alpha$, is less than $\alpha$. If $\alpha^{*}<\alpha$, the argument of Theorem 6 is followed closely, and the ordinals less than $\alpha^{*}$ take over the part of the natural numbers. If $\alpha^{*}=\alpha$, then a slight modification is necessary; instead of preserving values of $\{\varepsilon\}^{A}(\beta)$ for all $\beta<\alpha^{*}$, we preserve $\{\varepsilon\}^{A}(\beta)$ for all $\beta<\varepsilon$, and make use of the repetition inherent in any standard enumeration of the functions weakly $\alpha$-recursive in $A$. Thus we obtain a non- $\alpha$-recursive, $\alpha$-recursively enumerable set $A$ with the property that any function weakly $\alpha$-recursive in $A$, when restricted to an $\alpha$-finite set, is an $\alpha$-finite partial function.

5. The nonexistence of maximal sets. Let $\alpha$ be admissible. We say an $\alpha$-recursively enumerable set $M$ is maximal if $c M$ is not bounded by any ordinal less than $\alpha$ 
and if for each $\alpha$-recursively enumerable set $R$, either $R-M$ or $c R$ is bounded by some ordinal less than $\alpha$. Clearly, a maximal set is unbounded. The maximal set construction of [7] shows that if $\alpha^{*}=\omega$, then a maximal, $\alpha$-recursively enumerable set exists. It follows that there exist uncountably many, countable, admissible $\alpha$ such that a maximal, $\alpha$-recursively enumerable set exists. (In fact, it was shown in [7] that there exists a metarecursively enumerable set $M$ with unbounded complement such that for any metarecursively enumerable $R$, either $R-M$ or $c R$ is finite.) The purpose of the present section is to give an example of an important theorem about recursively enumerable sets which does not generalize to arbitrary admissible ordinals.

THEOREM 10. There exists a countable, admissible ordinal $\alpha$ such that no $\alpha$-recursively enumerable set is maximal.

Proof. First we show that no maximal, aleph-one-recursively enumerable set exists. Assume that $V=L$. Let $f$ be a one-one, aleph-one-recursive function which assigns an aleph-one-finite set of natural numbers to each countable ordinal; the equations defining $f$ merely specify some diagonal procedure. Let $g$ be the usual one-one function which assigns a member of the closed unit interval to each set of natural numbers. Then $h=g \circ f$ can be viewed as an aleph-one-recursive function, which associates a real number with each countable ordinal. Suppose $M$ is a maximal, aleph-one-recursively enumerable set. Let $I_{1}, I_{2}, I_{3}, \cdots$ be the usual enumeration of all open subintervals of the unit interval with rational endpoints. For each $n$, let $J_{n}=\left\{\gamma \mid h(\gamma) \in I_{n}\right\}$; then $J_{n}$ is aleph-one-recursively enumerable if a natural $h$ is chosen; in fact, $J_{n}$ is aleph-one-recursive uniformly in $n$. For each $n, J_{n}-M$ or $c J_{n}-M$ is countable; let $K_{n}$ be $J_{n}-M$ if $J_{n}-M$ is countable, and let $K_{n}$ be $c J_{n}-M$ otherwise. Let $K=\bigcup\left\{K_{n} \mid n<\omega\right\}$, and $N=c M-K$. Then for each $n, N \cap J_{n}=\varnothing$ or $N \cap c J_{n}=\varnothing$. Let $\alpha, \beta \in N$ such that $h(\alpha)<h(\beta)$, and $r_{1}, r_{2}$ be rational numbers such that $h(\alpha)<r_{1} \& h(\beta)<r_{2}$. Let $I_{m}=\left(r_{1}, r_{2}\right)$. Then $\beta \in J_{m}, \alpha \notin J_{m}, N \cap J_{m} \neq \varnothing$, and $N \cap c J_{m} \neq \varnothing$. (Of course, the aleph-one-recursive enumerability of $M$ is irrelevant.)

Let $P$ be a countable initial segment of the constructible sets such that $P$ is a model of set theory and $V=L$. The above argument holds in $P$. Let $\gamma$ be the countable ordinal which plays the part of aleph-one in $P$. The notion of admissibility is absolute, so $\gamma$ is admissible, not only in the sense of $P$, but in actuality. There is no maximal $\gamma$-recursively enumerable set in $P$, so there is none anywhere, since all $\gamma$-recursively enumerable sets are in $P$. The existence of $P$ cannot be proved in set theory, but $P$ can safely be replaced by $Q$, a model for finitely many axioms of set theory and $V=L$ strong enough to carry out the above argument; and the existence of $Q$ can be proved in set theory.

We do not know if there exists an uncountable, admissible ordinal $\alpha$ such that a maximal, $\alpha$-recursively enumerable set exists. We conjecture that it is possible to give an elementary description of an ordinal satisfying Theorem 10 . 
Note that the argument of Theorem 10 shows there does not exist any maximal CA (complementary analytic) set of real numbers. (Call a CA set $M$ maximal if its complement is uncountable and if for each CA set $R \supseteq M$, either $R-M$ is countable or $c R$ is countable.) This fact combined with the existence of maximal $\Pi_{1}^{1}$ sets provides strong evidence for the following assertion: the analogy between recursively enumerable sets and $\Pi_{1}^{1}$ sets is much stronger than the analog between $\Pi_{1}^{1}$ sets and CA sets. We will give other evidence in [13]. Of course, one is free to say that the above notion of maximality has no place in the theory of CA sets. The argument of Theorem 10 still works if one adopts the following definition: call a CA set $M$ maximal if its complement is not Borel and if for each CA set $R \supseteq M$, either $R-M$ is Borel or $c R$ is Borel.

6. Post's problem for admissible ordinals. Kripke [8] has obtained a solution of Post's problem for a large class of admissible ordinals. We give a solution of Post's problem which holds for every admissible $\alpha$, but we leave open the structure of the upper semilattice of degrees of $\alpha$-recursively enumerable sets.

THEOREM 11. For each admissible ordinal $\alpha$, there exist $\alpha$-recursively enumerable sets $A$ and $B$ such that $A$ is not $\alpha-r e c u r s i v e$ in $B$ and $B$ is not $\alpha$-recursive.

Proof. The introductory remarks of $\$ 3$ readily generalize to an arbitrary admissible ordinal $\alpha$; the natural numbers are replaced by the ordinals less than $\alpha^{*}$, the projectum of $\alpha$. Thus we have a T-predicate whose definition is:

$$
T_{1}^{1}(B, \varepsilon, \alpha, \sigma) \leftrightarrow t(\varepsilon, \sigma)=\langle\varepsilon, M, N, \alpha, \gamma\rangle \& M \subseteq B \& N \subseteq c B,
$$

where $\varepsilon<\alpha^{*}, B$ is a set of ordinals less than $\alpha$, and $M$ and $N$ are $\alpha$-finite sets; $t(\varepsilon, \sigma)$ is a special $\alpha$-recursive function which indexes "deductions." $T_{1}{ }^{1}(B, \varepsilon, \alpha, \sigma)$ says: $\varepsilon$ is the Gödel number of a finite system of equations $E$ and $t(\varepsilon, \sigma)$ is the Gödel number of an $\alpha$-finite "deduction" from $E$ and $B$, whose last line gives the value $\gamma$ of some function for argument $\alpha . U(\varepsilon, \sigma)$ is an $\alpha$-recursive function such that if $t(\varepsilon, \sigma)=\langle\varepsilon, M, N, \alpha, \gamma\rangle$, then $U(\varepsilon, \sigma)=\gamma$. Two useful partial functions are:

$$
\begin{aligned}
& \{\varepsilon\}_{\sigma}^{B}(\alpha)=\gamma \leftrightarrow T_{1}^{1}(B, \varepsilon, \alpha, \sigma) \& U(\varepsilon, \sigma)=\gamma ; \\
& \{\varepsilon\}^{B}(\alpha)=\gamma \leftrightarrow(E \sigma)\left(\{\varepsilon\}_{\sigma}^{B}(\alpha)=\gamma\right) \&(\sigma)\left(\{\varepsilon\}_{\sigma}^{B}(\alpha) \text { is defined } \rightarrow U(\varepsilon, \sigma)=\gamma\right) .
\end{aligned}
$$

If $A$ is weakly $\alpha$-recursively in $B$, then for some $\varepsilon<\alpha^{*}$, we have $(\gamma)\left(a(\gamma)=\{\varepsilon\}^{B}(\gamma)\right)$, where $a(\gamma)$ is the characteristic function of $A$.

Let $\left\{R_{\gamma} \mid \gamma<\alpha^{*}\right\}$ be a standard simultaneous enumeration of all the $\alpha$-recursively enumerable sets. Let $R_{\gamma}^{\sigma}$ be the set of all members of $R_{\gamma}$ enumerated prior to stage $\sigma$ of the simultaneous enumeration. Let $r(\sigma)$ be an $\alpha$-recursive function whose range is bounded by $\alpha^{*}$ such that for each $\varepsilon<\alpha^{*}$, the set $\{\sigma \mid r(\sigma)=\varepsilon\}$ is not bounded by any ordinal less than $\alpha$. 
We simultaneously define four $\alpha$-recursive functions, $A(\sigma), B(\sigma), d(\varepsilon, \sigma)$ and $K(\varepsilon, \sigma)$ by induction on $\sigma$.

Stage $\sigma=0 . A(o)=B(o)=\varnothing, d(\varepsilon, o)=\varepsilon$, and $K(\varepsilon, o)=0$ for all $\varepsilon<\alpha^{*}$.

Stage $\sigma>0$. Let $\delta=r(\delta), A\left(\sigma^{*}\right)=\bigcup\{A(\rho) \mid \rho<\sigma\}$, and $B\left(\sigma^{*}\right)=\bigcup\left\{B(\rho) \mid<\sigma^{*}\right\}$. Let $K\left(\varepsilon, \sigma^{*}\right)=$ least upper bound of $\{K(\varepsilon, \rho) \mid \rho<\sigma\}$ and $d\left(\varepsilon, \sigma^{*}\right)=$ least upper bound of $\{d(\varepsilon, \rho) \mid \rho<\sigma\}$.

If $R_{\delta}^{\sigma} \cap B\left(\sigma^{*}\right)=\varnothing$, and if there is a $\rho$ such that $\rho \in R_{\delta}^{\sigma} \&(\varepsilon)_{\varepsilon<\delta}\left(\rho>K\left(\varepsilon, \sigma^{*}\right)\right) \& \rho$ greater than double any member of $\bigcup\left\{R_{r(\tau)}^{\tau} \cap\left(B(\tau)-B\left(\tau^{*}\right)\right) \mid \tau<\sigma \& r(\tau)<\delta\right\}$, then add the least such $\rho$ to $B\left(\sigma^{*}\right)$ to obtain $B(\sigma)$; otherwise, $B(\sigma)=B\left(\sigma^{*}\right)$. We define $d(\varepsilon, \sigma)$ for all $\varepsilon<\alpha^{*}$ :

$$
\begin{aligned}
d(\varepsilon, \sigma)= & d\left(\varepsilon, \sigma^{*}\right) \text { if } \varepsilon<\delta \text { or if } B(\sigma)=B\left(\sigma^{*}\right) ; \\
d(\varepsilon, \sigma)= & \mu x(\tau)(\tau<\varepsilon \rightarrow x>d(\tau, \sigma)) \\
\& x> & \left.d\left(\varepsilon, \sigma^{*}\right)+\text { least upper bound of } A\left(\sigma^{*}\right)+1\right) \text { if } \\
& \varepsilon \geqq \delta \& B(\sigma) \neq B\left(\sigma^{*}\right) .
\end{aligned}
$$

Now we consider $\{\delta\}_{\gamma}^{B\left(\sigma^{*}\right)}(d(\delta, \sigma))$ for all $\gamma<\sigma$. Suppose $d(\delta, \sigma) \notin A\left(\sigma^{*}\right)$ and there is a $\gamma<\sigma$ such that $\{\delta\}_{\gamma}^{B\left({ }^{*}\right)}(d(\delta, \sigma))=1$; let $\tau$ be the least such $\gamma$. Define $A(\sigma)=A\left(\sigma^{*}\right) \cup\{d(\delta, \sigma)\}$. Let $K(\delta, \sigma)=K\left(\delta, \sigma^{*}\right)+$ least upper bound of $N(\delta, \tau)$, and let $K(\varepsilon, \sigma)=K\left(\varepsilon, \sigma^{*}\right)$ for all $\varepsilon \neq \delta$ and $<\alpha^{*}$. If our supposition is false, let $A(\sigma)=A\left(\sigma^{*}\right)$ and $K(\varepsilon, \sigma)=K\left(\varepsilon, \sigma^{*}\right)$ for all $\varepsilon<\alpha^{*}$.

LEMMA 11. For each $\delta<\alpha^{*}$, there exists a $\tau$ such that for all $\varepsilon \leqq \delta, d(\varepsilon, \tau)=d(\varepsilon, \sigma)$ for all $\sigma \geqq \tau$.

Proof. Fix $\delta<\alpha^{*}$. At the beginning of stage $\sigma>0$, we consider adding an element to $B$ for the sake of $R_{\gamma}$; this operation occurs at most once before the requirement that $B\left(\sigma^{*}\right) \cap R_{\gamma}^{\sigma}=\varnothing$. Let $S$ be the set of all $\gamma \leqq \varepsilon$ such that at some stage $\sigma$, a number of $R_{\gamma}^{\sigma}$, where $\gamma=r(\sigma)$, is added to $B . S$ is $\alpha$-recursively enumerable and is bounded by $\delta<\alpha^{*}$. It follows that $S$ is $\alpha$-finite. Let $\tau$ be so large that all members of $S$ have been enumerated prior to stage $\tau$.

LEMMA 12. $B$ is not $\alpha$-recursive.

Proof. First we show $c B$ is unbounded. Fix $\gamma<\alpha$ and suppose $\{\beta \mid \gamma \leqq \beta<\alpha\} \subseteq B$. Let $\alpha_{0}$ be an infinite ordinal $>\gamma$ such that $\alpha_{0} \in B(\sigma)-B\left(\sigma^{*}\right)$ and $\alpha_{0}>$ every member of $B\left(\sigma^{*}\right)$. For each $n \geqq 0$, let $\alpha_{n+1}$ be the first member of $B$ to be enumerated after $\alpha_{n}$ with the property that $\alpha_{0}<\alpha_{n+1}<2 \alpha_{n}$; our supposition concerning $\gamma$ implies $\alpha_{n}$ is well-defined for all $n<\omega$. Let $\delta(n)$ be such that $\alpha_{n}$ is added to $B$ at stage $\sigma(n)$ for the sake of $R_{\delta(n)}$; in short, $r(\sigma(n))=\delta(n)$. Clearly, any element added to $B$ for the sake of $R_{\delta}$ at stage $\sigma$, where $\delta>\delta(n)$ and $\sigma>\sigma(n)$, must be greater than $2 \alpha_{n}$; consequently, $\delta(n+1)<\delta(n)$ for all $n$, a contradiction.

Now fix $\delta$ and suppose $R_{\delta}$ is unbounded; we show $R_{\delta} \neq c B$. Let $\tau$ be as in the 
statement of Lemma 11 . Let $\beta \geqq \tau$ be such that $\lim _{\sigma} d(\varepsilon, \sigma) \in A \leftrightarrow \lim _{\sigma} d(\varepsilon, \sigma) \in A\left(\beta^{*}\right)$ for all $\varepsilon \leqq \delta$. The existence of $\beta$ follows from the properties of $\alpha^{*}$ and $\tau$ and the fact that $\delta<\alpha^{*}$. But then $K(\varepsilon, \beta)=K(\varepsilon, \sigma)$ for all $\varepsilon \leqq \delta$ and $\sigma \geqq \beta$. Let $\rho$ be such that $\rho \in R_{\delta},(\varepsilon)_{c<\delta}\left(\rho>\lim _{\sigma} K(\varepsilon, \sigma)\right)$, and $\rho$ is greater than double any member of $\bigcup\left\{R_{r(\gamma)}^{\gamma} \cap\left(B(\gamma)-B\left(\gamma^{*}\right)\right) \mid r(\gamma)<\delta\right\}$; the existence of $\rho$ follows from the nature of $\alpha^{*}$. Then $B$ must contain some element of $R$.

LEMMA 13. $A$ is not weakly $\alpha$-recursive in $B$.

Proof. Fix $\delta$. Suppose $\{\delta\}^{B}(\gamma)$ is defined for all $\gamma<\alpha$. Let $d=\lim _{\sigma} d(\delta, \sigma) \in A$. For some $\sigma$, we must have $d=d(\delta, \sigma) \notin A\left(\sigma^{*}\right)$ and $\{\delta\}_{\tau}^{B\left(\sigma^{*}\right)}(d)=1$ for some $\tau<\sigma$. Also, $K(\delta, \sigma) \geqq$ every member of $N(\delta, \tau)$. We claim $\{\delta\}^{B}(d)=1$; it is sufficient to show $N(\delta, \tau) \subseteq c B$. If some element of $N(\delta, \tau)$ is put in $B$ at stage $\rho>\sigma$, then $r(\rho)=\beta \leqq \delta$ and $d(\delta, \rho) \neq d\left(\delta, \rho^{*}\right)$. But $\rho>\sigma$ and $\lambda \gamma \mid d(\delta, \gamma)$ is a nondecreasing function of $\gamma$, so $d(\delta, \rho)=d\left(\delta, \rho^{*}\right)$.

Now suppose $d \notin A$. Then $\{\varepsilon\}^{B}(d) \neq 1$, since otherwise the second half of stage $\sigma$ would eventually compel us to put $d$ in $A$.

The proof of Theorem 11 used only one combinatorial fact concerning all admissible ordinals; namely, an $\alpha$-recursively enumerable set bounded by an ordinal less than $\alpha^{*}$, the projectum of $\alpha$, is $\alpha$-finite. If $\alpha^{*}<\alpha$, then $\alpha^{*}$ behaves somewhat like $\omega$ does in metarecursion theory. If $\alpha^{*}=\alpha$, then all bounded, $\alpha$-recursively enumerable sets are $\alpha$-finite, and it is possible to initiate some familiar arguments of ordinary recursion theory. But we are concealing many combinatorial dificulties. The proof of Theorem 8 works because at most one attempt is made to satisfy each requirement of the form: if $R_{e}$ is unbounded, then $R_{e} \cap B \neq \varnothing$. We have not found any combinatorial trick for attacking all admissible ordinals with priority arguments that permit more than one attempt to satisfy any requirement.

7. Open questions and conjectures. What follows is not a complete list of all interesting questions concerning the topics of this paper, but is, hopefully, a selection.

Q1. If $\alpha$ is admissible, is $\alpha^{*}$, the projectum of $\alpha$, admissible? This question was suggested by Kripke, who has shown that $\alpha^{*}$ must be the limit of admissible ordinals $\left({ }^{3}\right)$.

Q2. Do there exist $\alpha$-recursively enumerable subsets of $\alpha^{*}$ of incomparable $\alpha$-degrees? We think yes. The answer to Q1 might be of some help.

Q3. Do there exist $\alpha$-recursively enumerable sets such that neither is computable from the other by means of Kripke's equation calculus. We think yes.

Q4. For what $\alpha$ do maximal, $\alpha$-recursively enumerable sets exist? In particular, is there a maximal, $\alpha$-recursively enumerable set for some uncountable $\alpha$ ?

If $A$ is weakly metarecursive in $B$, then $A=\{e\}^{B}$ for some $e$ as described at

(3) Kripke has recently shown the answer to Q1 is yes. 
the beginning of $\S 3$. The reduction procedure used to compute $A$ from $B$ can be identified with the metarecursively enumerable set $\left\{t(e, \sigma) \mid \sigma<\omega_{1}\right\}$. A typical value of $t(e, \sigma)$ is $\langle e, M, N, \alpha, \gamma\rangle$; if $M \subseteq B$ and $N \subseteq c B$, then $\{e\}^{B}(\alpha)=\gamma$, where $M$ and $N$ are metafinite sets. We say $e$ is intrinsically consistent, if for all $\alpha, \sigma_{1}$ and $\sigma_{2}$, if $t\left(e, \sigma_{1}\right)=\left\langle e, M_{1}, N_{1}, \alpha, \gamma_{1}\right\rangle, \quad t\left(e, \sigma_{2}\right)=\left\langle e, M_{2}, N_{2}, \alpha, \gamma_{2}\right\rangle$, and $\left(M_{1} \cup M_{2}\right) \cap\left(N_{1} \cup N_{2}\right)=\varnothing$, then $\gamma_{1}=\gamma_{2}$.

Q5. For each $e$, does there exist an intrinsically consistent $e^{*}$ such that $\{e\}^{A} \simeq\left\{e^{*}\right\}^{A}$ for all $A$ ? We think not. In ordinary recursion theory the answer is yes, since we can require that $M$ and $N$ define a finite, initial segment of a characteristic function rather than an arbitrary, basic neighborhood.

Q6. How does one define the jump operator for sets of recursive ordinals? A satisfactory definition should have the property that if $A$ is metarecursive in $B$, then the jump of $A$ is metarecursive in the jump of $B$. An answer to Q5 should help here.

Q7. Does there exist a metarecursive function $f$ such that for all $e, R_{f(e)}$ is regular and has the same metadegree as $R_{e}$ ? The proof of Theorem 1 does not provide such an $f$, but we think it exists. An answer to Q8 might help.

Q8. If $A$ is a nonregular, metarecursively enumerable set, does there exist a $\Pi_{1}^{1}$ set of the same metadegree as $A$ ? We conjecture yes $\left({ }^{4}\right)$.

In[13] we will give axioms for recursion theory in the sense that one gives axioms for group theory. Any object satisfying our axioms will be called a recursion system and will consist of an initial segment of the ordinaals and some partial functions on that segment and into it. It will be seen that most of the results of this paper hold for arbitrary recursion systems and that the notion of regular, hyperregular set (inspired by Kreisel's notion of subgeneric set) is the key to the classification of recursion systems.

\section{REFERENCES}

1. J. C. E. Dekker, A theorem on hypersimple sets, Proc. Amer. Math. Soc. 5 (1954), 791-796.

2. G. Driscoll, Contributions to metarecursion theory, $\mathrm{Ph}$. D. Thesis, Cornell University, Ithaca, N. Y., 1965.

3. R. M. Friedberg, Two recursively enumerable sets of incomparable degrees of unsolvability, Proc. Nat. Acad. Sci. U.S.A. 43 (1957), 236-238.

4. —_, Three theorems on recursive enumeration, J. Symbolic Logic 23 (1958), 309-316.

5. S. C. Kleene, Introduction to metamathematics, Van Nostrand, New York, 1952.

6. G. Kreisel, Model-theoretic invariants, Theory of Models Symposium, Berkeley, Calif., (to appear).

7. G. Kreisel and G. E. Sacks, Metarecursive sets, J. Symbolic Logic 31 (1966), 1-21.

8. S. Kripke, Transfinite recursions on admissible ordinals. I, II (abstracts), J. Symbolic Logic 29 (1964), 161-162.

9. - Admissible ordinals and the analytic hierarchy (abstract), J. Symbolic Logic 29 (1964), 162.

(4) Note added in proof. We can show the answer to Q8 is yes. 
10. E. L. Post, Recursively enumerable sets of positive integers and their decision procedures, Bull. Amer. Math. Soc. 50 (1944), 284-316.

11. G. E. Sacks, Degrees of unsolvability, Annals of Mathematics Studies No. 55, Princeton Univ. Press, Princeton, N. J., 1963.

12. - The recursively enumerable degrees are dense, Ann. of Math. 80(1964), 300-312.

13. _- Axioms for recursion theory, (to appear).

14. C. Spector, Recursive well-orderings, J. Symbolic Logic 20 (1955), 151-163.

\section{CORNELl UNIVERSITY,} ITHACA, NeW YORK 